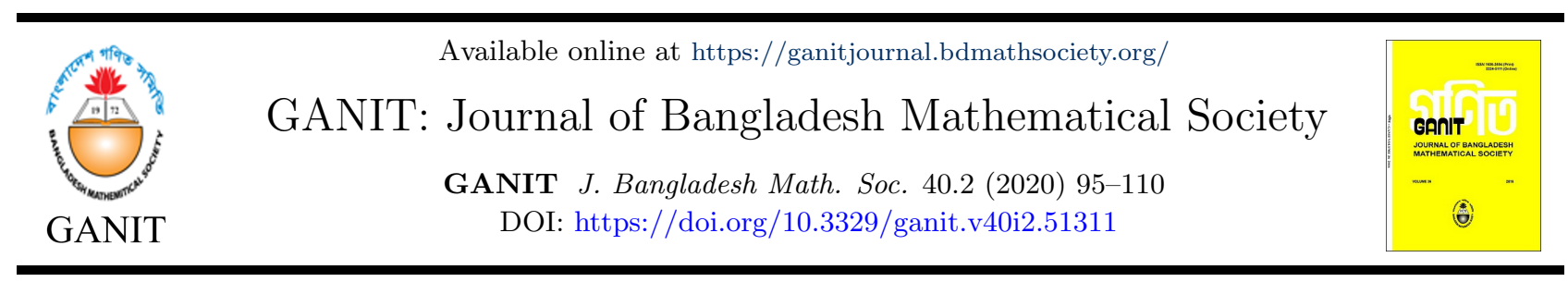

\title{
Lyapunov Stability Analysis of a Competition Model with Crowding Effects
}

\author{
Md. Kamrujjaman*a ${ }^{*}$ Kamrun Nahar Keya ${ }^{\mathrm{b}}$, and Md Shafiqul Islam ${ }^{\mathrm{c}}$

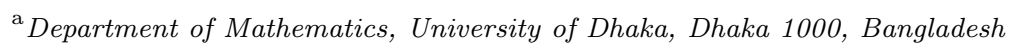 \\ ${ }^{\mathrm{b}}$ Department of Science and Humanities, Military Institute of Science and Technology, Mirpur Cantonment, Dhaka 1216, \\ Bangladesh \\ ${ }^{\mathrm{c}}$ School of Mathematical and Computational Sciences, University of Prince Edward Island, Charlottetown, PE, Canada
}

\begin{abstract}
The present study is connected to the analysis of a nonlinear system that covered a wide range of mathematical biology in terms of competition, cooperation, and symbiosis interactions between two species. We focus on how populations change their densities when two different species follow the non-symmetric logistic growth laws. We have investigated the stability of the corresponding densities of population, and to control the convergence of solutions by proper choice of interacting constant and periodic parameters. It shows the effect of crowding tolerance on both species. It will show that there exists an infinite number of coexistence solutions if the resource distributions are identical for both populations. If the carrying capacity of the first species exceeds the rest one, then eventually the second population drops down to extinction. The results are presented studying the Lyapunov functional, phase portraits, and in a series of numerical examples.
\end{abstract}

(c) 2020 Published by Bangladesh Mathematical Society

Received: July 27, 2020 Accepted: October 26, 2020 Published Online: January 15, 2021

Keywords: Lyapunov functional; stability; competition; crowding effects; phase portraits; numerical solutions.

AMS Subject Classifications 2020: 92D25, 35K57 (primary), 35K61, 37N25.

\section{Introduction}

The study of competition among different species is a prevailing feature in ecology. Different biological considerations are known to develop two-species competition models using different growth functions. Competition arises whenever at least two species strive for a goal that cannot be shared, where one's gain is the other's loss. When they are only affected by their populations, then this type of competition is defined as intraspecific competition [1]. When resources are limited, several species may depend on these resources. Thus, each of the species competes with the others to gain access to the resources and this type of competition is known as interspecific competition [2, 3, 4]. In interspecific competition species less suited to compete for the resources may die out unless they adapt by character dislocation. In the literature, the well developed and most popular growth laws were established in $[5,6,7,8,9,10]$. In this paper, we develop a basic competition model based on

\footnotetext{
*Corresponding author. E-mail address: kamrujjaman@du.ac.bd
} 
two different species either intraspecific or interspecific competition with two different growth rates and their carrying capacities are non-identical. Taking into account of two different species, we consider the following system of nonlinear differential equations with initial conditions:

$$
\left\{\begin{array}{l}
\frac{d u}{d t}=r_{1}(t) u(t)\left(1-\frac{u(t)+v(t)}{k_{1}(t)}\right), t>0 \\
\frac{d v}{d t}=r_{2}(t) u(t)\left(1-\frac{u(t)+v(t)}{k_{2}(t)}\right), t>0 \\
u\left(t_{0}\right)=u_{0}>0, \quad v\left(t_{0}\right)=v_{0}>0
\end{array}\right.
$$

This system of equations (1.1) consists of functions that are smooth and continuous. Here $u$ and $v$ denote the population density of two species and $k_{1}, k_{2}$ represent their respective carrying capacity. Similarly, $r_{1}$ and $r_{2}$ are the specific growth rates of $u$ and $v$ respectively. It is assumed that both functions defined in (1.1) are smooth enough, e.g., $C^{1+\alpha}, 0<\alpha<1$ over time domain. The density of $u$ and $v$ is always positive since we assume that population density is not negative. Henceforth (1.1) is an initial value problem with conditions $u\left(t_{0}\right)>0$ and $v\left(t_{0}\right)>0$. Since the carrying capacity and growth rates of the two populations are distinct, the dynamics will show the crowding effect behavior which is an important observation in the study. It is also remarked that the carrying capacities and intrinsic growth rates are time-periodic as well as constant. If carrying capacity is higher in a habitat then the movement of the population will increase in the niche compared to other habitats; biologically the dynamics of the crowding effect will arise. We will discuss here how growth rates change due to the change in carrying capacity and also give some illustrations on the stability of the population. Among various methods for analyzing the stability of nonlinear systems, we use here a well-known method of Lyapunov functional.

Instead of logistic growth, let us consider the following function

$$
f(t, u, k, M)=r(t) u(t)\left(1-\frac{u(t)}{k(t)}\right)\left(\frac{u(t)}{M(t)}-1\right), t>0,
$$

where, $r$ is the intrinsic growth rate, and $k$ is the carrying capacity for population $u$. The function $M$ is the sparse function and shows the Allee effect $[11,12,13]$. If $0<M<k$ then there is a weak Allee effect while the strong Allee effect is visible for $M<0<k$. If we consider the mathematical model introducing the function defined in (1.2), both crowding and Allee effect study can be observed. It is noted that the crowding out in ecology is a result of competition by parasites within a host for finite resources. Typically, the severity of this effect increases with increasing numbers of parasites within a host and manifests in reduced body size and thus fitness. A strong Allee effect moves the species to extinction even for a weak Allee effect in many cases.

It is also noted that we introduced the linearization techniques [14] and linear differential equation theory to analyze the steady states of our considered model. Often, mathematical models of real-world phenomena are formulated in terms of systems of nonlinear differential equations [15, 16], which can be difficult to solve explicitly. To overcome this barrier, we take a qualitative approach to the analysis of solutions to nonlinear systems by making phase portraits and using stability analysis [17, 18, 19, 20]. We demonstrate these techniques in the analysis of an individual problem in the area of nonlinear differential equations. The model is originally motivated by population models in mathematical biology when solutions are required to be non-negative, but the differential equations can be understood outside of this traditional scope of population models.

In mathematical modeling of ecology, the asymptotic behavior of the solutions of two species interacting population has been central to understanding the coexistence and spatial dissociation of two species [21, 22, 23]. In the weak competition case, the positive stationary solution $(u, v)$ is globally asymptotically stable independent of the diffusion coefficients. Materially, there exists a Lyapunov functional which allows for a long-time asymptotic analysis [24]. The recent study has been proven that the directed dispersal organism has evolutionary advantages to designing its own habitat for a competition coupled species model [25, 26].

We represent the stability of equilibria of the model in various parameters; at least two important constants or periodic parameters that make the difference in the logistic growth model. It is found that if the competition favors the latter species, there is still a range of parameters for which there is coexistence. Numerical investigation shows the long term behavior of the complete model that characterizes the population densities on several levels. Throughout the entire study, we come to a conclusion of the stability of different competing species.

The rest of the paper is organized in the following way. In Section 2, we discuss the basic study of Lyapunov stability and Lyapunov functional. Section 3 presents the process of finding Lyapunov functional and techniques of stability analysis through the Lyapunov function for a general system. In Section 4, we discuss the local 
and global stability of equilibriums with phase portrait. A number of examples are presented in Section 5 for applications. Finally, Section 6 concludes the summary of the results and future discussions.

\section{Preliminaries}

In this section, we introduce some auxiliary results which are available in the literature [2, 7, 27, 28], and these features will be used throughout the paper.

\subsection{Lyapunov stability}

Linear stability analysis tells us how a system behaves near an equilibrium point. It does not however tell us anything about what happens farther away from equilibrium. Phase-plane analysis combined with linear stability analysis can generally give us a full picture of the dynamics, but things become much more difficult in higher-dimensional spaces. In this section, we consider a technique due to Lyapunov which can be used to determine the stability of an equilibrium point [10, 16, 29]. This method forms the basis of much of modern nonlinear control theory and also provides a theoretical justification for using local linear control techniques. Lyapunov stability is an intuitive approach to analyzing the stability and convergence of dynamic systems without explicitly computing the solutions of their differential equations.

To discuss Lyapunov stability for nonlinear equations we need to demonstrate some important definitions which are given below:

Definition 1. [27] A point $x^{*}$ is said to be an equilibrium point of

$$
\frac{d x}{d t}=f(t, x)
$$

at time $t_{0}$ if for all $t \geq t_{0}, f\left(t, x^{*}\right)=0$.

Definition 2. [27] The equilibrium $x=0$ of (2.1) is stable if, for each time $t_{0}$, and for every constant $R>0$, there exists some $r\left(R, t_{0}\right)$ such that

$$
\left\|x\left(t_{0}\right)\right\|<r \text { implies, }\|x(t)\|<R \text { for all } t \geq t_{0} .
$$

It is uniformly stable if $r$ is independent of $t_{0}$. The equilibrium is unstable if it is not stable.

Definition 3. [27] The equilibrium $x=0$ of (2.1) is asymptotically stable if: (a) it is stable, and (b) for each time $t_{0}$ there exists some $r\left(t_{0}\right)>0$ such that

$$
\left\|x\left(t_{0}\right)\right\|<r \Rightarrow\|x(t)\| \rightarrow 0 \text { as } t \rightarrow \infty
$$

It is uniformly asymptotically stable if it is asymptotically stable and both $r$ is independent of $t_{0}$.

\subsection{Lyapunov's method}

In many cases, it is possible to determine whether an equilibrium of a nonlinear system is locally stable by examining the stability of the linear approximation to the nonlinear dynamics about the equilibrium point. This approach is known as Lyapunov's method.

Theorem 1. [27, 28] Let $\dot{x}=f(x), f\left(x^{*}\right)=0$ where $\dot{x}=\frac{d x}{d t}$ and $x^{*}$ is in the interior of $\Omega \subset \mathbb{R}^{n}$. Assume that $V: \Omega \rightarrow \mathbb{R}$ is a function and

(i) $V\left(x^{*}\right)=0$.

(ii) $V(x)>0$ for all $x \in \Omega, x \neq x^{*}$. 
(iii) $\dot{V}(x) \leq 0$ along all trajectories of the system in $\Omega$.

(iv) $\dot{V}<0$ for all $x \in \Omega, x \neq x^{*}$.

If (i)-(iii) is satisfing then $x^{*}$ is said to be locally stable and if (iv) is also satisfied then $x^{*}$ is said to be locally asymptotically stable.

A function that fulfills condition (i)-(iii) is called Lyapunov function.

\subsection{Hartman-Grobman theorem}

The Hartman-Grobman theorem is another important result in the local qualitative theory of ordinary differential equations (ODEs). The theorem shows that $\dot{x}=f(x)$ with $f(0)=0$ and its linearized system $\dot{x}=\mathcal{D} f(x) x$ have the same qualitative structures near a hyperbolic equilibrium.

Definition 4. [27] Let $A$ and $B$ be subsets of $\mathbb{R}^{n}$. A homomorphism $A$ onto $B$ is a continuous one-to-one map $H: A \rightarrow B$ such that $H^{-1}: B \rightarrow A$. The two sets $A$ and $B$ are called homomorphic or topologically equivalent. Consider

$$
\dot{x}=f(x)
$$

Where $x=0$ is a hyperbolic equilibrium. The linearized system

$$
\dot{x}=A x
$$

where $A=\mathcal{D} f(0)$.

Theorem 2. [27] If $x=0$ is a hyperbolic equilibrium (2.2) and (2.3), then there exists a homomorphism $H$ of an open set $U$ containing the origin onto an open set $V$ containing the origin such that for each $x_{0} \in U$, there exists an open interval $I_{0} \subset \mathbb{R}$ containing the origin such that for all $t \in I_{0}$

$$
H \circ \phi_{t}\left(x_{0}\right)=e^{A t} H\left(x_{0}\right)
$$

\section{Lyapunov Functional and Techniques of Stability Analysis}

\subsection{Construction of Lyapunov function}

We can construct a Lyapunov function by two methods-one is a variable gradient method, and the other is Krasovskii's generalized method. In this paper, we grab the first method for analyzing the solution of the proposed model.

Assume that the gradient of the Lyapunov function $V(x)$ is known up to some parameters.

$$
\begin{gathered}
\nabla V(x)=\left[V_{1}, \cdots \cdots, V_{n}\right]^{T}=\left[\frac{\partial V}{\partial x_{1}}, \cdots \cdots, \frac{\partial V}{\partial x_{n}}\right]^{T} \\
\nabla V_{i}=\sum_{j=1}^{n} h_{i j} x_{j}, i=1, \cdots \cdots, n
\end{gathered}
$$

Using the curl conditions $\frac{\partial^{2} V}{\partial x_{i} \partial x_{j}}=\frac{\partial \nabla V_{i}}{\partial x_{j}}=\frac{\partial \nabla V_{j}}{\partial x_{i}}$ simplify the coefficients $h_{i j}$ for $i, j=1, \cdots, n$ and hence obtain $\dot{V}(x) \equiv \frac{\partial V}{\partial x}$. Then integrate it to obtain $V(x)$ as shown below

$$
V(x)=\int_{0}^{x} \nabla V(x) d x
$$

Executing this method, we have to keep in mind that the value of $h_{i j}, i, j=1, \cdots, n$ should be chosen so that $V(x)$ is positive definite and $\dot{V}(x)$ is negative definite. 


\subsection{Techniques for solving nonlinear systems using eigenvalues}

Consider a nonlinear system of differential equations using dummy variables $x$ and $y$ :

$$
\left\{\begin{array}{l}
x^{\prime}=f(x, y) \\
y^{\prime}=g(x, y)
\end{array}\right.
$$

Where $f$ and $g$ are functions of two variables $x=x(t)$ and $y=y(t)$ such that $f$ and $g$ are not both linear functions of $x$ and $y$. It is also noted that the notation $x^{\prime}=\frac{d x}{d t}$ and $y^{\prime}=\frac{d y}{d t}$.

Unlike a linear system, a nonlinear system could have none, one, two, three, or any number of critical points. However, the critical points are found setting $x^{\prime}=y^{\prime}=0$, and solve the resulting system

$$
\begin{aligned}
& f(x, y)=0 \\
& g(x, y)=0
\end{aligned}
$$

And every solution of this system of algebraic equations is an equilibrium point of the given system (3.1). Since there might be multiple equilibrium points present in the phase portrait, each trajectory could be influenced by more than one equilibrium point. These results have a much more chaotic appearance of the phase portrait [29]. We will approximate the behavior of the nearby trajectories using the linearizations of $f$ and $g$. The technique converts the nonlinear system into a linear system whose phase portrait approximates the local behavior of the original nonlinear system near the equilibrium point.

Consider the Taylor series expansion about the equilibrium point $(x, y)=(\alpha, \beta)$ and we have

$$
\begin{aligned}
x^{\prime}=f(x, y) & \approx f(\alpha, \beta)+f_{x}(\alpha, \beta)(x-\alpha)+f_{y}(\alpha, \beta)(y-\beta) \\
y^{\prime}=g(x, y) & \approx g(\alpha, \beta)+g_{x}(\alpha, \beta)(x-\alpha)+g_{y}(\alpha, \beta)(y-\beta)
\end{aligned}
$$

Since $f(\alpha, \beta)=0=g(\alpha, \beta)$, the above linearizations become

$$
\begin{aligned}
x^{\prime} & =f(x, y)=f_{x}(\alpha, \beta)(x-\alpha)+f_{y}(\alpha, \beta)(y-\beta) \\
y^{\prime} & =g(x, y)=g_{x}(\alpha, \beta)(x-\alpha)+g_{y}(\alpha, \beta)(y-\beta)
\end{aligned}
$$

Now it is a homogeneous linear system with a coefficient matrix

$$
A=\left(\begin{array}{ll}
f_{x}(\alpha, \beta) & f_{y}(\alpha, \beta) \\
g_{x}(\alpha, \beta) & g_{y}(\alpha, \beta)
\end{array}\right)
$$

Above matrix is calculated by putting $x=\alpha$, and $y=\beta$ into the matrix of first partial derivatives

$$
J(x, y)=\left(\begin{array}{ll}
f_{x} & f_{y} \\
g_{x} & g_{y}
\end{array}\right)
$$

This matrix $J(x, y)$ is often called the Jacobian matrix. It needs to be calculated once for each nonlinear system. For each critical point of the system, all we need to do is to compute the coefficient matrix of the linearized system about the equilibrium point $(x, y)=(\alpha, \beta)$ and then use its eigenvalues to determine the stability.

\section{Local and Global Stability Analysis}

\subsection{Linearization of the System}

First, we have to linearize the system for further analysis. Rewrite the functions of equation (1.1) to find the Lyapunov function as

$$
\left\{\begin{array}{l}
r_{1}(t) u(t)\left(1-\frac{u(t)+v(t)}{k_{1}(t)}\right)=f(u, v) \\
r_{2}(t) u(t)\left(1-\frac{u(t)+v(t)}{k_{2}(t)}\right)=g(u, v)
\end{array}\right.
$$


To find the linearization matrices at the equilibria, we differentiate the equations of (4.1) and then obtain,

$$
\begin{aligned}
& \frac{\partial f}{\partial u}=\frac{r_{1}}{k_{1}}\left(k_{1}-2 u-v\right) \\
& \frac{\partial f}{\partial v}=-\frac{r_{1}}{k_{1}} u \\
& \frac{\partial g}{\partial u}=-\frac{r_{2}}{k_{2}} v \\
& \frac{\partial g}{\partial v}=\frac{r_{2}}{k_{2}}\left(k_{2}-u-2 v\right)
\end{aligned}
$$

These partial functions produce the linearization matrix $\nabla V(u, v)$, and hence

$$
\nabla V(u, v)=\left(\begin{array}{ll}
\frac{\partial f}{\partial u} & \frac{\partial f}{\partial v} \\
\frac{\partial g}{\partial u} & \frac{\partial g}{\partial v}
\end{array}\right)\left(\begin{array}{l}
u \\
v
\end{array}\right)
$$

and finally which yields

$$
\nabla V(u, v)=\left(\begin{array}{cc}
\frac{r_{1}}{k_{1}}\left(k_{1}-2 u-v\right) & -\frac{r_{1}}{k_{1}} u \\
-\frac{r_{2}}{k_{2}} v & \frac{r_{2}}{k_{2}}\left(k_{2}-u-2 v\right)
\end{array}\right)\left(\begin{array}{l}
u \\
v
\end{array}\right)
$$

Substituting the coordinates of the equilibrium points $(0,0),\left(k_{1}, 0\right),\left(0, k_{2}\right)$ and $\left(u^{*}, v^{*}\right)$ into this formula, we obtain the linearization matrices to discuss the stability analysis of our main problem (1.1).

In the following section, we will consider all the equilibrium points to find stability or instability conditions.

\subsection{Steady states analysis}

Trivial equilibrium $(0,0)$ : At the equilibrium point $(u, v)=(0,0)$, four partial derivatives are $\frac{\partial f}{\partial u}=r_{1}$, $\frac{\partial f}{\partial v}=0, \frac{\partial g}{\partial u}=0, \frac{\partial g}{\partial v}=r_{2}$. Inserting these values in (4.2), we execute $\nabla(u, v)$ :

$$
\nabla V(u, v)=\left(\begin{array}{cc}
r_{1} & 0 \\
0 & r_{2}
\end{array}\right)\left(\begin{array}{l}
u \\
v
\end{array}\right)=\left(\begin{array}{l}
r_{1} u \\
r_{2} v
\end{array}\right)
$$

Integrating the gradient function yields the Lyapunov function $V$ such that

$$
\begin{aligned}
V(u, v) & =\int_{0}^{u} r_{1} x d x+\int_{0}^{v} r_{2} y d y \\
& =r_{1} \frac{u^{2}}{2}+r_{2} \frac{v^{2}}{2}>0, r_{1}>0, r_{2}>0
\end{aligned}
$$

Let us now differentiate the previous function $V(u, v)$, and we obtain

$$
\begin{aligned}
\frac{\partial V}{\partial t} & =r_{1} u \frac{d u}{d t}+r_{2} v \frac{d v}{d t} \\
& =\frac{r_{1}^{2}}{k_{1}} u^{2}\left(k_{1}-u-v\right)+\frac{r_{2}^{2}}{k_{2}} v^{2}\left(k_{2}-u-v\right), \text { using }(1.1)
\end{aligned}
$$

If $\frac{\partial V}{\partial t}>0$ by considering $u>0, v>0$ as Lyapunov stability conditions, it can be concluded that the equilibrium point $(0,0)$ is locally unstable. 
Due to the graphical analysis, the linearization matrix at $(0,0)$ is

$$
A_{(0,0)}=\left(\begin{array}{cc}
r_{1} & 0 \\
0 & r_{2}
\end{array}\right)
$$

We consider both $r_{1}>r_{2}$ and $r_{1}<r_{2}$. Let us first consider $r_{1}=2$ and $r_{2}=1$ which gives eigenvalues $\lambda_{1}=2, \lambda_{2}=1$ and the corresponding eigenvectors

$$
\left(\begin{array}{l}
1 \\
0
\end{array}\right) \text { and }\left(\begin{array}{l}
0 \\
1
\end{array}\right)
$$

respectively.

Here both eigenvalues are real and positive which concludes that the equilibrium point $(0,0)$ is a repeller. Biologically, when both of the species are present in the same ecological circumference they will repel each other and leave the sub-domain of the habitat until other factors are considered. Similar conclusions can be made by considering $r_{1}=2$ and $r_{2}=3$. At this critical point, the system is always unstable which is showing in Figure 4.1 .
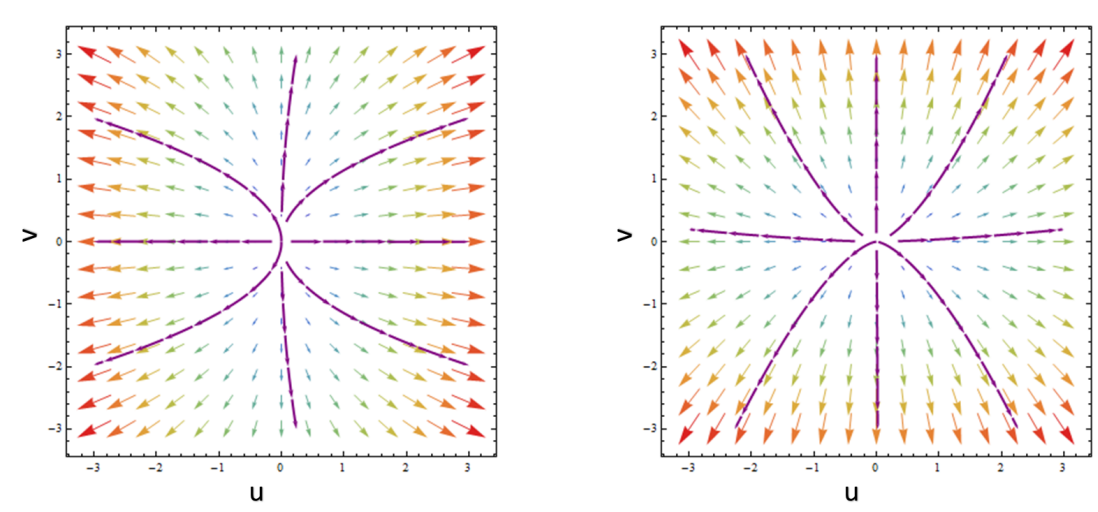

Figure 4.1: Phase portrait of the linear system of $(1.1)$ near $(0,0)$ for (left) $r_{1}=2, r_{2}=1$ and (right) $r_{1}=2$, $r_{2}=3$.

Semi-trivial equilibrium $\left(k_{1}, 0\right)$ : At the equilibrium point $(u, v)=\left(k_{1}, 0\right)$, the partial derivatives are $\frac{\partial f}{\partial u}=$ $-r_{1}, \frac{\partial f}{\partial v}=-r_{1}, \frac{\partial g}{\partial u}=0, \frac{\partial g}{\partial v}=\frac{r_{2}}{k_{2}}\left(k_{2}-k_{1}\right)$. To execute $\nabla(u, v)$, substitute all partials in (4.2) and we obtain

$$
\nabla V(u, v)=\left(\begin{array}{cc}
-r_{1} & -r_{1} \\
0 & \frac{r_{2}}{k_{2}}\left(k_{2}-k_{1}\right)
\end{array}\right)\left(\begin{array}{l}
u \\
v
\end{array}\right)=\left(\begin{array}{c}
-r_{1} u-r_{1} v \\
\frac{r_{2}}{k_{2}}\left(k_{2}-k_{1}\right) v
\end{array}\right)
$$

On integration, we will find Lyapunov function $V$ given below,

$$
\begin{aligned}
V(u, v) & =\int_{0}^{u}\left(-r_{1} x\right) d x+\int_{0}^{v} \frac{r_{2}}{k_{2}}\left(k_{2}-k_{1}\right) y d y \\
& =-r_{1} \frac{u^{2}}{2}+\frac{r_{2}}{k_{2}}\left(k_{2}-k_{1}\right) \frac{v^{2}}{2}
\end{aligned}
$$

For stability, we must find the criterion such that $V$ is always positive. Assume $r_{1}=r_{2}$ and hence $V>0$ which implies

$$
-r_{1} \frac{u^{2}}{2}+\frac{r_{2}}{k_{2}}\left(k_{2}-k_{1}\right) \frac{v^{2}}{2}>0
$$




$$
\begin{aligned}
& \Rightarrow-u^{2} k_{2}+\left(k_{2}-k_{1}\right) v^{2}>0, \text { since } r_{1}>0, r_{2}>0 \\
& \Rightarrow \frac{\left(k_{2}-k_{1}\right)}{k_{2}}>\frac{u^{2}}{v^{2}}>0 .
\end{aligned}
$$

which implies that $k_{2}>k_{1}$. Now, differentiating the function $V$, we have

$$
\begin{aligned}
\frac{\partial V}{\partial t} & =-r_{1} u \frac{d u}{d t}+\frac{r_{2}}{k_{2}}\left(k_{2}-k_{1}\right) v \frac{d v}{d t} \\
& =-\frac{r_{1}^{2}}{k_{1}} u^{2}\left(k_{1}-u-v\right)+\left(\frac{r_{2}}{k_{2}}\right)^{2}\left(k_{2}-k_{1}\right) v^{2}\left(k_{2}-u-v\right)
\end{aligned}
$$

For satisfying the Lyapunov condition, we must consider $\frac{\partial V}{\partial t}<0$. Hence either

$$
\begin{aligned}
& -\frac{r_{1}^{2}}{k_{1}}\left(k_{1} u^{2}-u^{3}-v u^{2}\right)<0 \\
& \Rightarrow k_{1}>u+v, r_{1}>0, k_{1}>0
\end{aligned}
$$

Or

$$
\begin{aligned}
& \left(\frac{r_{2}}{k_{2}}\right)^{2}\left(k_{2}-k_{1}\right) v^{2}\left(k_{2}-u-v\right)<0 \\
& \Rightarrow 0<k_{2}<u+v \text { if } k_{2}>k_{1}
\end{aligned}
$$

However if $k_{1}>k_{2}$ then we obtain the following inequalities

$$
k_{2}>u+v
$$

Considering conditions, it can be clarified that for the case $k_{1}>k_{2}$, the equilibrium point $\left(k_{1}, 0\right)$ is locally as well as globally asymptotically stable independently of initial densities, see Figure 5.3(right).

Remark 1. It is noticed that both $u>0$ and $v>0$ throughout this section to satisfy all the inequalities of stability analysis.

For the shake of graphical analysis, consider the linearization matrix at $\left(k_{1}, 0\right)$ :

$$
A_{\left(k_{1}, 0\right)}=\left(\begin{array}{cc}
-r_{1} & -r_{1} \\
0 & \frac{r_{2}}{k_{2}}\left(k_{2}-k_{1}\right)
\end{array}\right)
$$

where corresponding eigenvalues are $\lambda_{1}=-r_{1}$ and $\lambda_{2}=\frac{r_{2}}{k_{2}}\left(k_{2}-k_{1}\right)$. Here $\lambda_{2}>0$ if $k_{2}>k_{1}$ and $\lambda_{2}<0$ for $k_{2}<k_{1}$. Initially we consider $r_{1}=r_{2}=1$ and $k_{2}>k_{1}$, where $k_{1}=1, k_{2}=2$ and we obtain the eigenvalues $\lambda_{1}=-1$ and $\lambda_{2}=0.5$ and corresponding eigenvectors are

$$
\left(\begin{array}{l}
1 \\
0
\end{array}\right) \text { and }\left(\begin{array}{c}
-0.83077 \\
0.556666
\end{array}\right)
$$

respectively. Since both eigenvalues are negative, we can summarize the critical point $\left(k_{1}, 0\right)$ is an asymptotically stable point shown in Figure 4.2 (right). So, in this case, as the species, $v$ is extinct from the given ecological territory, whereas the species $u$ is the sole winner.

Semi-trivial equilibrium $\left(0, k_{2}\right)$ : At the equilibrium point $(u, v)=\left(0, k_{2}\right)$, the corresponding partial derivatives are $\frac{\partial f}{\partial u}=\frac{r_{1}}{k_{1}}\left(k_{1}-k_{2}\right), \frac{\partial f}{\partial v}=0, \frac{\partial g}{\partial u}=-r_{2}, \frac{\partial g}{\partial v}=-r_{2}$. Substituting these values in (4.2), one can implement $\nabla V(u, v)$ as:

$$
\nabla V(u, v)=\left(\begin{array}{cc}
\frac{r_{1}}{k_{1}}\left(k_{1}-k_{2}\right) & 0 \\
-r_{2} & -r_{2}
\end{array}\right)\left(\begin{array}{c}
u \\
v
\end{array}\right)=\left(\begin{array}{c}
\frac{r_{1}}{k_{1}}\left(k_{1}-k_{2}\right) u \\
-r_{2} u-r_{2} v
\end{array}\right)
$$



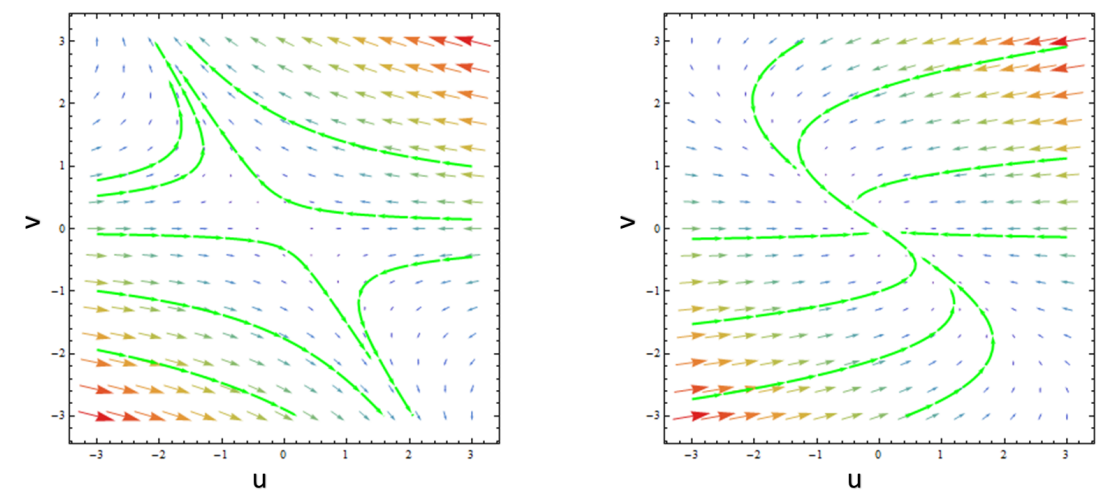

Figure 4.2: Phase portrait of the linear system of (1.1) for $r_{1}=r_{2}=1$ and (left) $k_{2}>k_{1}, k_{1}=1, k_{2}=2$ and (right) $k_{1}>k_{2}, k_{1}=4, k_{2}=3$.

On integration provides the suitable Lyapunov function $V$ such that

$$
\begin{aligned}
V(u, v) & =\int_{0}^{u} \frac{r_{1}}{k_{1}}\left(k_{1}-k_{2}\right) x d x+\int_{0}^{v}\left(-r_{2} y\right) d y \\
& =\frac{r_{1}}{k_{1}}\left(k_{1}-k_{2}\right) \frac{u^{2}}{2}-r_{2} \frac{v^{2}}{2}
\end{aligned}
$$

Assume $r_{1}=r_{2}$, and consider the stability criteria $V>0$ which due to the following inequalities

$$
\begin{aligned}
& \frac{r_{1}}{k_{1}}\left(k_{1}-k_{2}\right) \frac{u^{2}}{2}-r_{2} \frac{v^{2}}{2}>0 \\
& \Rightarrow \frac{\left(k_{1}-k_{2}\right)}{k_{1}}>\frac{v^{2}}{u^{2}} \\
& \Rightarrow k_{1}>k_{2} \text {, while } u>0, v>0 .
\end{aligned}
$$

Now differentiating $V$, we have

$$
\begin{aligned}
\frac{\partial V}{\partial t} & =\frac{r_{1}}{k_{1}}\left(k_{1}-k_{2}\right) u \frac{d u}{d t}-r_{2} v \frac{d v}{d t} \\
& =\left(\frac{r_{1}^{2}}{k_{1}}\right)^{2}\left(k_{1}-k_{2}\right)\left(k_{1} u^{2}-u^{3}-v u^{2}\right)-\frac{r_{2}}{k_{2}}\left(k_{2} v^{2}-u v^{2}-v^{3}\right)
\end{aligned}
$$

For satisfying the Lyapunov condition, let us consider $\frac{\partial V}{\partial t}<0$. Therefore, either

$$
\begin{aligned}
& \left(\frac{r_{1}^{2}}{k_{1}}\right)^{2}\left(k_{1}-k_{2}\right)\left(k_{1} u^{2}-u^{3}-v u^{2}\right)<0 \\
& \Rightarrow 0<k_{1}<\frac{u^{3}+v u^{2}}{u^{2}}
\end{aligned}
$$

Or

$$
\begin{aligned}
& -\frac{r_{2}}{k_{2}}\left(k_{2} v^{2}-u v^{2}-v^{3}\right)<0 \\
& \Rightarrow k_{2}>\frac{u v^{2}+v^{3}}{v^{2}}
\end{aligned}
$$


Considering these two inequalities, we can conclude that if $k_{2}>k_{1}$ then the equilibrium point $\left(0, k_{2}\right)$ is both locally and globally asymptotically stable for any non-negative and non-trivial initial values, see Figure 5.3(left).

To study the graphical analysis via phase plane, let us check the linearization matrix at $\left(0, k_{2}\right)$ such that

$$
A_{\left(0, k_{2}\right)}=\left(\begin{array}{cc}
\frac{r_{1}}{k_{1}}\left(k_{1}-k_{2}\right) & 0 \\
-r_{2} & -r_{2}
\end{array}\right)
$$

where $\lambda_{1}=r_{1} / k_{1}\left(k_{1}-k_{2}\right)$ and $\lambda_{2}=-r_{2}$ are respective eigenvalues. The eigenvalues $\lambda_{1}>0$ if $k_{1}>k_{2}$ and $\lambda_{1}<0$ if $k_{2}>k_{1}$. It is also seen that the eigenvalue $\lambda_{2}$ is always negative. For instantaneous example, consider $r_{1}=r_{2}$ and $k_{1}>k_{2}$; specifically let us assume that $k_{1}=3, k_{2}=2$ and $r_{1}=r_{2}=1$ and the respective eigenvalues are $\lambda_{1}=-1$ and $\lambda_{2}=0.33$ with corresponding eigenvectors:

$$
\left(\begin{array}{l}
0 \\
1
\end{array}\right) \text { and }\left(\begin{array}{c}
0.799278 \\
-0.600961
\end{array}\right)
$$

Two eigenvalues of the matrix $A_{\left(0, k_{2}\right)}$ are both real and have opposite signs. Thus the equilibrium $\left(0, k_{2}\right)$ is an unstable saddle point as shown in Figure 4.3 (left).

Similarly, we consider $r_{1}=r_{2}=1$ and $k_{2}>k_{1}$. Put $k_{1}=2, k_{2}=3$ and in this case the eigenvalues are $\lambda_{1}=-1$ and $\lambda_{2}=-0.5$ and corresponding eigenvectors are

$$
\left(\begin{array}{l}
0 \\
1
\end{array}\right) \text { and }\left(\begin{array}{c}
0.447214 \\
-0.894427
\end{array}\right)
$$

respectively. Since both eigenvalues $\lambda_{1}$ and $\lambda_{2}$ are negative, the critical point $\left(0, k_{2}\right)$ is an asymptotically stable node as depicted in Figure 4.3 (right). Therefore, if the second species, $v$ has more accessibility to the resources then the first population, $u$ goes to extinction.
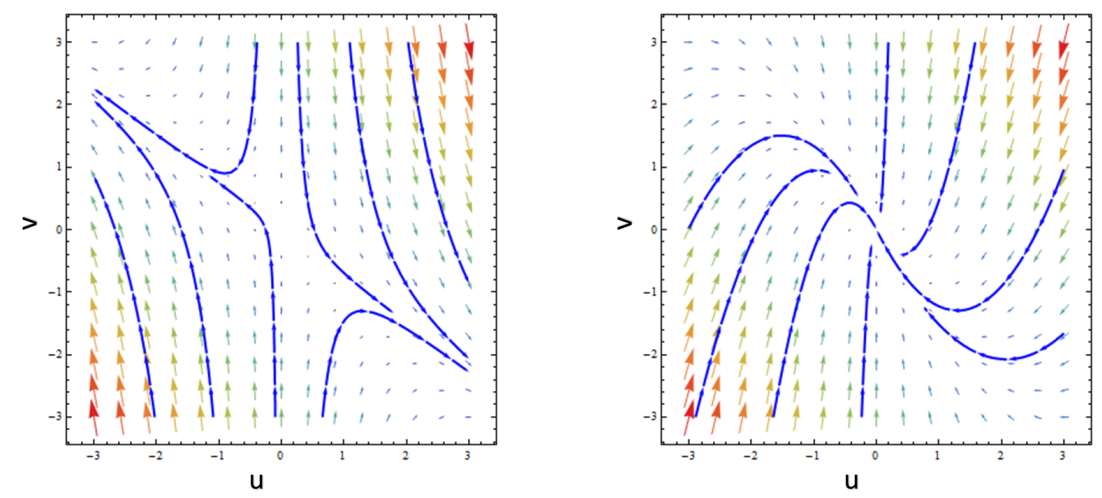

Figure 4.3: Phase portrait of the linear system of (1.1) for $r_{1}=r_{2}=1$ with (left) $k_{1}>k_{2}, k_{1}=3, k_{2}=2$ and (right) $k_{2}>k_{1}, k_{1}=2, k_{2}=3$.

Coexistence equilibrium $\left(u^{*}, v^{*}\right)$ : Since we do not know the exact coexistence equilibrium point, so it is not possible to find the general Lyapunov function at coexistence point $\left(u^{*}, v^{*}\right)$. If we place some assumptions regarding parameters, then we can formulate a local Lyapunov function or region.

Let $k_{1}=k_{2}=k$ in some non-empty open domain of nature. Then we have the equilibrium point $\left(u^{*}, v^{*}\right)=$ $(\alpha k,(1-\alpha) k)$, with $\alpha \in(0,1)$. Then at equilibrium $(\alpha k,(1-\alpha) k)$, the corresponding partial derivatives are $\frac{\partial f}{\partial u}=-r_{1} \alpha, \frac{\partial f}{\partial v}=-r_{1} \alpha, \frac{\partial g}{\partial u}=-r_{2}(1-\alpha), \frac{\partial g}{\partial v}=-r_{2}(1-\alpha)$. By putting these values in (4.2), the gradient of $V(u, v)$ is defined as follows

$$
\nabla V(u, v)=\left(\begin{array}{cc}
-r_{1} \alpha & -r_{1} \alpha \\
-r_{2}(1-\alpha) & -r_{2}(1-\alpha)
\end{array}\right)\left(\begin{array}{l}
u \\
v
\end{array}\right)
$$




$$
=\left(\begin{array}{c}
-r_{1} \alpha(u+v) \\
-r_{2}(1-\alpha)(u+v)
\end{array}\right)
$$

Integrating $\nabla V$ provides the following Lyapunov function $V$ such that

$$
\begin{aligned}
V(u, v) & =\int_{0}^{u}\left(-r_{1} \alpha\right) x d x+\int_{0}^{v}\left(-r_{2}(1-\alpha)\right) y d y \\
& =-r_{1} \alpha \frac{u^{2}}{2}-r_{2}(1-\alpha) \frac{v^{2}}{2}
\end{aligned}
$$

Regarding the stability analysis, we must find the criterion such that $V$ is always positive. Assume $r_{1}=r_{2}=r$ and $V>0$ implies

$$
\begin{aligned}
& -r \alpha \frac{u^{2}}{2}-r(1-\alpha) \frac{v^{2}}{2}>0 \\
& \Rightarrow \frac{-\alpha}{(1-\alpha)}>\frac{v^{2}}{u^{2}} \\
& \Rightarrow \frac{-\alpha}{(1-\alpha)}>0, \text { while } u>0, v>0 \\
& \Rightarrow 1-\alpha>0 \Rightarrow 0<\alpha<1
\end{aligned}
$$

So $V$ will be positive for $\alpha \in(0,1)$, which was our primary assumption of coexistence solution. To check the local or global stability of the coexistence solution, differentiate the function $V$, and we have

$$
\begin{aligned}
\frac{\partial V}{\partial t} & =-r_{1} \alpha u \frac{d u}{d t}-r_{2}(1-\alpha) v \frac{d v}{d t} \\
& =-\frac{r_{1}^{2}}{k} \alpha\left(k u^{2}-u^{3}-v u^{2}\right)-\frac{r_{2}^{2}}{k}(1-\alpha)\left(k v^{2}-u v^{2}-v^{3}\right)
\end{aligned}
$$

For satisfying the Lyapunov strategy, the necessary condition is $\frac{\partial V}{\partial t}<0$ and hence, either

Or

$$
\begin{aligned}
& -\frac{r_{1}^{2}}{k} \alpha\left(k u^{2}-u^{3}-v u^{2}\right)<0 \\
& \Rightarrow k>\frac{u^{3}+v u^{2}}{u^{2}}
\end{aligned}
$$

$$
\begin{aligned}
& -\frac{r_{2}^{2}}{k}(1-\alpha)\left(k v^{2}-u v^{2}-v^{3}\right)<0 \\
& \Rightarrow k>\frac{u v^{2}+v^{3}}{v^{2}}
\end{aligned}
$$

Considering these two conditions it can be illuminated that when $\alpha \in(0,1)$, the equilibrium point $(\alpha k,(1-\alpha) k)$ is globally asymptotically stable, see Figure 5.3(middle).

Consider the linearization matrix at $(\alpha k,(1-\alpha) k)$ such that

$$
A_{(\alpha k,(1-\alpha) k)}=\left(\begin{array}{cc}
-r_{1} \alpha & -r_{1} \alpha \\
-r_{2}(1-\alpha) & -r_{2}(1-\alpha)
\end{array}\right)
$$

where $\lambda_{1}=0$ and $\lambda_{2}=-\left(r_{1} \alpha+r_{2}(1-\alpha)\right)$ are respective eigenvalues. Since one eigenvalue is zero, so here we do not have any point attractor or a point from which it will be repelled.

Here $\lambda_{1}=0$ and since both $r_{1}, r_{2}>0$ and $\alpha \in(0,1)$ so $\lambda_{2}<0$, that is $\lambda_{2}$ is always negative. For instance, let us consider $r_{1}=r_{2}=1$ and the respective eigenvalues for $\alpha=0.8$ are $\lambda_{1}=0$ and $\lambda_{2}=-1$ and for these eigenvalues we have infinitesimal eigenvectors for different choice of $u$ or $v$.

Since eigenvalue $\lambda_{2}$ are negative, the critical point $(\alpha k,(1-\alpha) k)$ is an asymptotically stable node shown in Figure 4.4. Therefore, the coexistence of both species is reliable when the resources are equals to both. 

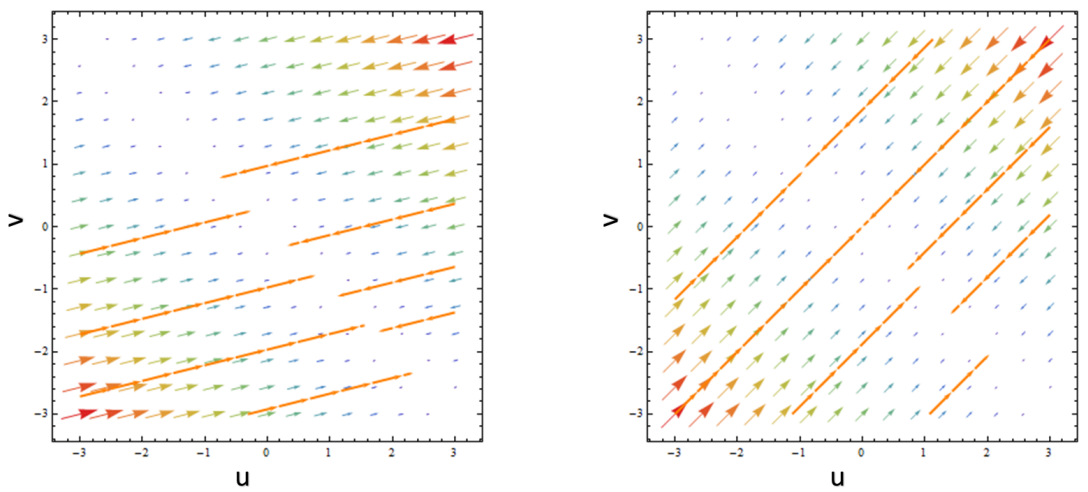

Figure 4.4: Phase portrait of the linear system of (1.1) for $r_{1}=r_{2}=1$ and $k_{1}=k_{2}=1$ with (left) $\alpha=0.8$, and (right) $\alpha=0.5$.

\section{$5 \quad$ Numerical Results and Discussions}

In this section, we discuss some examples of numerical solutions of the initial value problem (IVP) (1.1). The parameters like specific growth and resource function play a significant role to change population density.

Example 1. Consider the influence of carrying capacity on the density of two competing species $u$ and $v$ where intrinsic growth rates $r_{1}$ and $r_{2}$ of two species are considered to be identical. Assume $r_{1}=r_{2}=1.0$ and $k_{1}>k_{2}$. Here we fix the value of $k_{1}$ as $k_{1}=2+\sin (t)$ and varies $k_{2}=1.1,1.5$ and 1.7 .
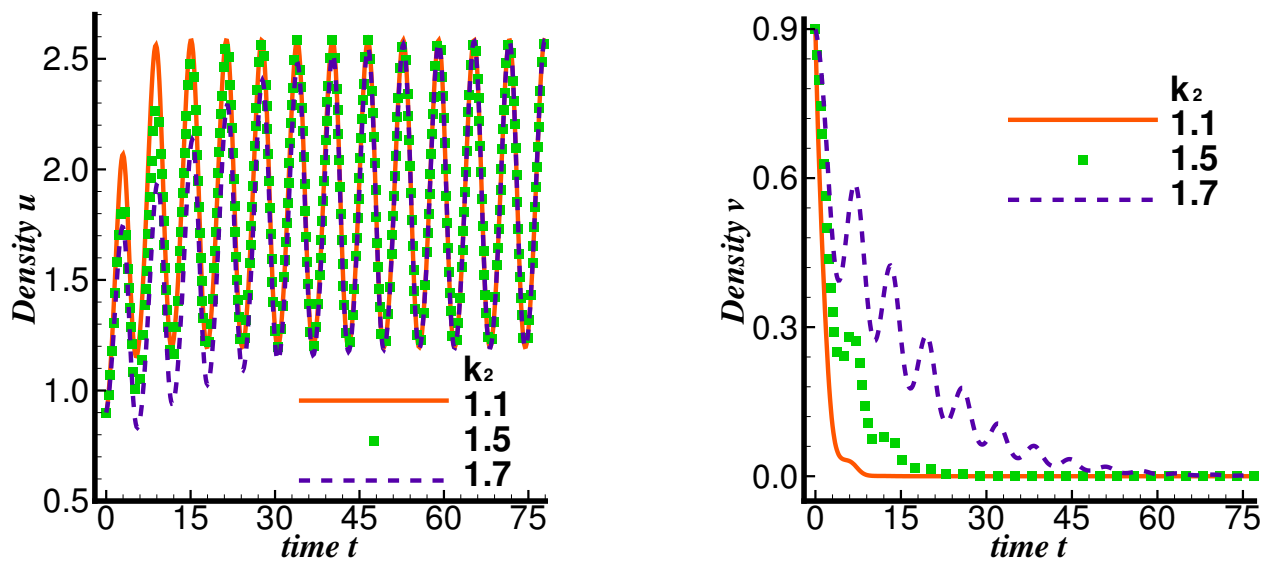

Figure 5.1: Numerical solutions of (1.1) for $r_{1}=r_{2}=1.0$ and $k_{1}=2.0+\sin (t), k_{2}=1.1,1.5$ and 1.7 with (left) the density of $u$, and (right) the density of $v$, where initial densities are $u_{0}=0.9$ and $v_{0}=0.9$.

Comparing the different values of $k_{2}$, there is an equilibrium with the increasing values of $u$, and decreasing values of $v$ as the carrying capacity of $u$ is higher than that of $v$ in a time domain and lower for a time sub-domain. When time is increasing, then the coexistence is not possible, the population with lower carrying capacity is repelling gradually.

As a result, the species $u$ survives, and species $v$ is going to extinct. See Figure 5.1 for graphical presentation.

Example 2. Consider the influence of periodic carrying capacities on the density of two competing species $u$ and $v$ where intrinsic growth rates $r_{1}, r_{2}$ of two species are considered to be same. Assume $r_{1}=r_{2}=1.0$ and $k_{2}>k_{1}$. Here we consider the value of $k_{2}$ as $k_{2}=2.5+\sin (t)$ while $k_{1}=1.1+\sin (t), 1.5+\sin (t)$ and $2.0+\sin (t)$. 
Considering these different values of $k_{1}$ there is an equilibrium with the decreasing values of $u$ and increasing values of $v$ as the carrying capacity of $u$ is considered to be lower than that of $v$, see Figure 5.2.
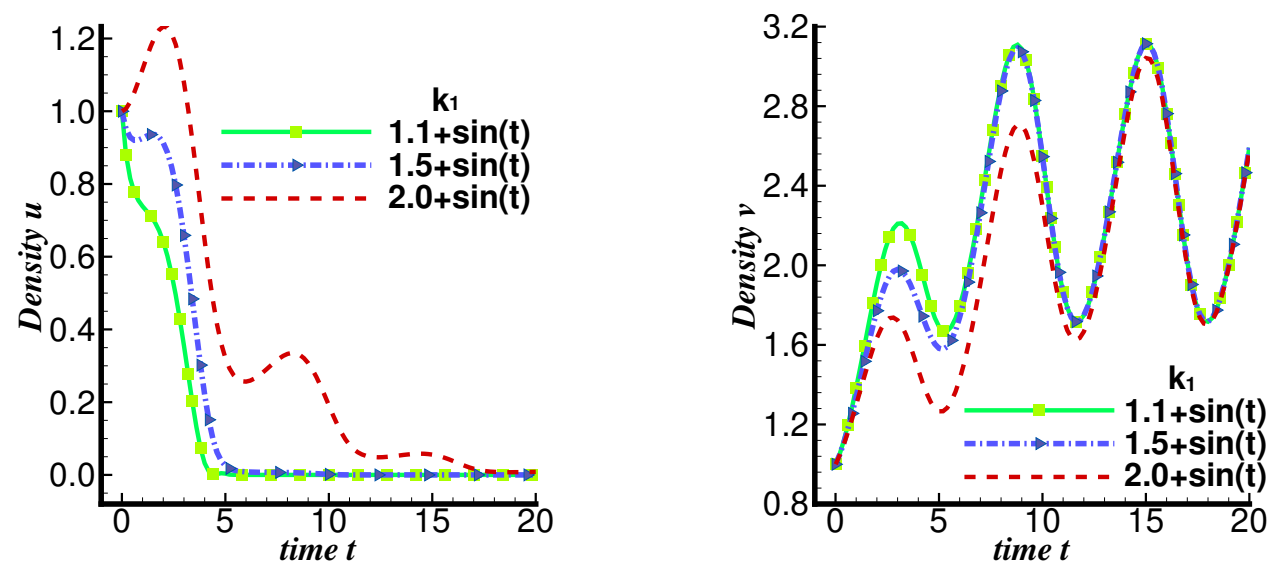

Figure 5.2: Numerical solutions of (1.1) for $r_{1}=r_{2}=1.0$ and $k_{1}=1.1+\sin (t), 1.5+\sin (t)$ and $2.0+\sin (t)$ when $k_{2}=2.5+\sin (t)$ with (left) the density of $u$, and (right) the density of $v$, where $\left(u_{0}, v_{0}\right)=(1.0,1.0)$.

Figure 5.2 is the case when the carrying capacity of $k_{2}$ has a higher value than $k_{1}$ in some non-empty open time domain. This situation will cause species u to extinction.

If we consider $k_{2}$ a little lower than the previous one and as far the time increases, the species $u$ repelled and $v$ survives periodically.

Example 3. Assume that growth rate of both species $u$ and $v$ are identical, that is $r_{1}=r_{2}$. Here we considered three cases, at first assume that carrying capacities are not same and $v$ has better environment than $u$, so $k_{2}>k_{1}$. In this case we let initial density of $u$ as $u_{0}=1.5$ and $v$ as $v_{0}=0.1$, then for $k_{1}=2$ and $k_{2}=4$ we see in first figure of Figure 5.3 that $u$ is in extinction and $v$ is converging to $k_{2}$.
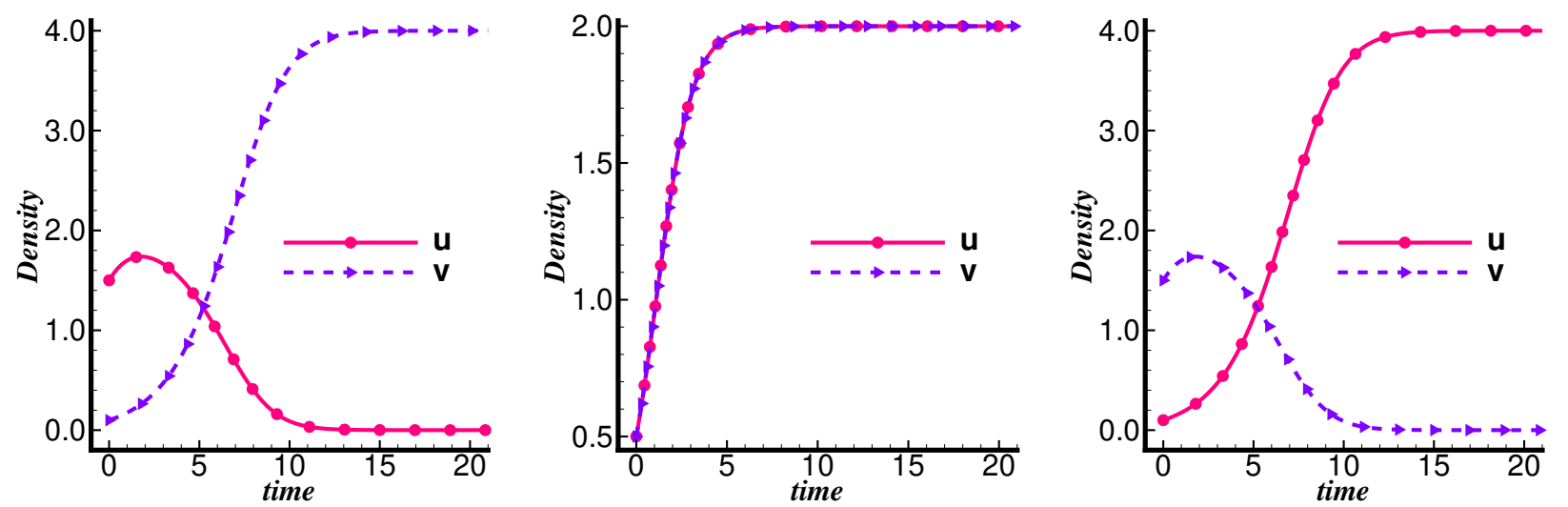

Figure 5.3: Numerical solutions of (1.1) for $r_{1}=r_{2}=1.0$ where in (left) $k_{1}=2.0, k_{2}=4.0$ with $\left(u_{0}, v_{0}\right)=$ $(1.5,0.1)$, in (middle) $k_{1}=k_{2}=4.0$ with $u_{0}=v_{0}=0.5$ and in (right) $k_{1}=4.0, k_{2}=2.0$ with $\left(u_{0}, v_{0}\right)=$ $(0.1,1.5)$.

In the second case, we let carrying capacities and initial densities are equal. In particular we choose $k_{1}=$ $k_{2}=4$ and $u_{0}=v_{0}=0.5$, we have coexistence and both species converges to same direction (see middle figure of Figure 5.3). 
Lastly we consider the contrary case of the first one. We assumed that $k_{1}>k_{2}$ and by choosing $k_{1}=4$, $k_{2}=2$ and initial densities as $\left(u_{0}, v_{0}\right)=(0.1,1.5)$ then in the last figure of Figure 5.3 shows that one species is in extinction and the another one is converging towards carrying capacity. For this properties species $v$ is in extinction and species $u$ converges to $k_{1}$.

Example 4. Consider the equal carrying capacities for both species; for example, $k_{1}=k_{2}=2.0+\sin (t)$. Here we show that the effects of intrinsic growth rates on the density of populations by varying growth rates for each species.
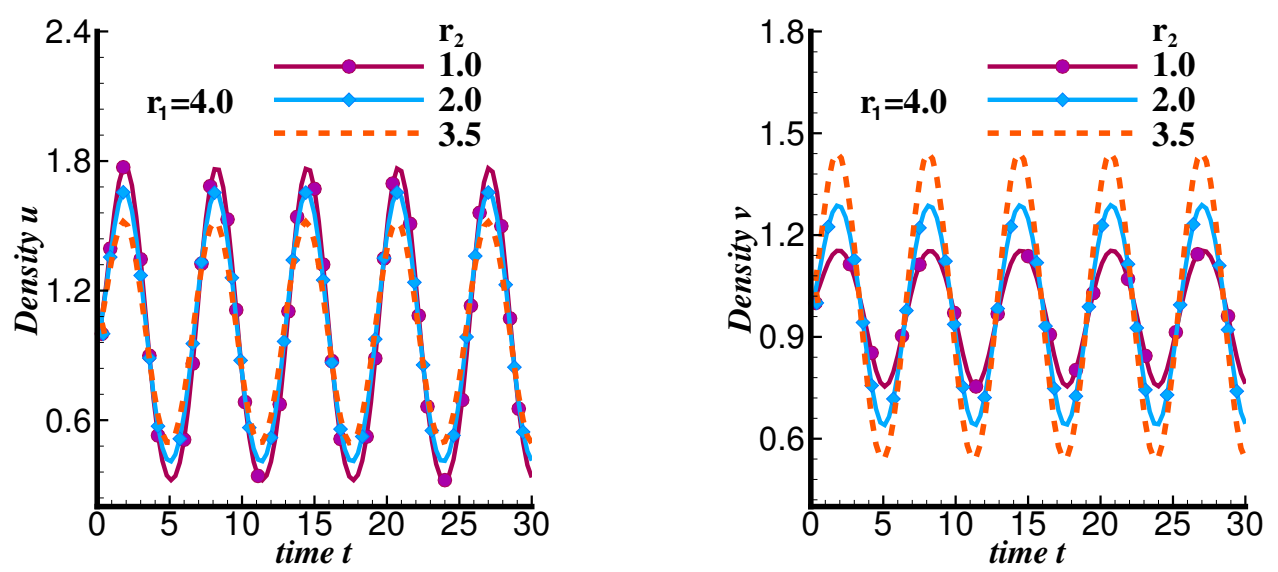

Figure 5.4: Numerical solutions of (1.1) for $k_{1}=k_{2}=2.0+\sin (t)$ and $r_{1}=4.0, r_{2}=1.0,2.0$ and 3.5 with (left) the density of $u$, and (right) the density of $v$, where initial densities are $u_{0}=v_{0}=1.0$.

If we fix $r_{1}=4.0$ and varies $r_{2}=1.0,2.0$ and 3.5, then we have a coexistence steady state which shows that the density of the first species will increase with a higher growth rate compared to the rest one, $v$, see Figure 5.4 .

Example 5. Consider the equal and time-periodic carrying capacity for both species, for example, $k_{1}=k_{2}=$ $4.0+\cos (t)$, and we want to observe the effects of specific growth rates.
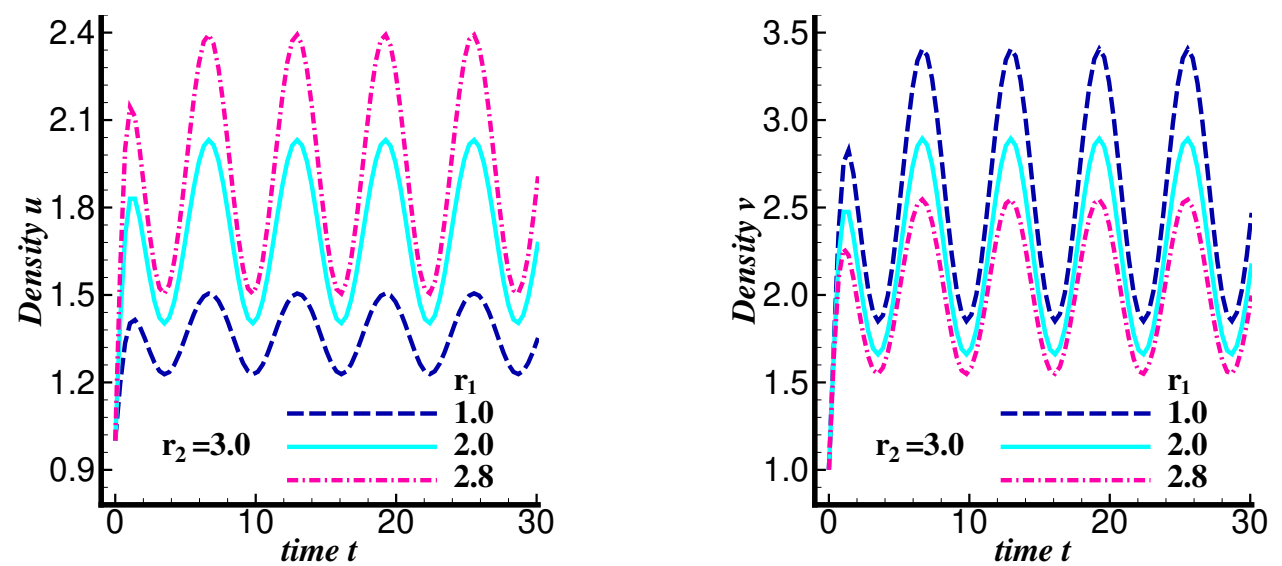

Figure 5.5: Numerical solutions of (1.1) for $k_{1}=k_{2}=4.0+\cos (t)$ and $r_{1}=1.0,2.0,2.8, r_{2}=3.0$ with (left) density of $u$, and (right) density of $v$, where $\left(u_{0}, v_{0}\right)=(1.0,1.0)$.

If we fix $r_{2}=3.0$ and consider $r_{1}=1.0,2.0$ and 2.8, then we have seen that if the growth rate of the first species is lower compared to the second one, the density $u$ of the first species decreases and larger values of $r_{1}$ leads to the increasing value of $u$ in the coexistence equilibrium. In this case, density $v$ of the second species is always increasing, see Figure 5.5. 


\section{Conclusions}

In this paper, we studied a two-species competition population model both analytically and numerically. The results were presented for local and global cases using phase diagrams and periodic functions. It was seen that in many cases it is possible to determine whether an equilibrium of a nonlinear system is locally stable or globally asymptotically stable simply by Lyapunov's theorem. This method relies on the observation that asymptotic stability is intimately linked to the existence of a Lyapunov function. The system is asymptotically stable means that the equilibrium state at the origin is asymptotically stable, if and only if the eigenvalues have negative real parts. By this stability analysis, we will get a clear conception about the growth and extinction of various competitive species. Numerical investigations both confirmed and completed the theoretical results while comparing the density of two different species. Here we have seen that the considered model suggests that carrying capacity plays an important role in the determination of the competition outcome between two species in a given ecological circumference. If the carrying capacity remains the same for two species, the population with a larger growth rate grows high and the population with a smaller growth rate decreases gradually. When growth rates are considered identical, the population with a larger carrying capacity has higher density and is the sole winner. Dynamical behavior of the system was investigated at equilibrium points. As one parameter is varied, the dynamics of the system near to this solution change the stability. Both analytically and numerically, the results showed that the model sensitively depends on the parameter values and initial conditions. For future studies, it would be interesting if one can study these models for particular species in any habitats.

\section{Acknowledgments}

The authors are grateful to the anonymous reviewers for their constructive suggestions to improve the quality of the paper significantly. The research was partially supported by TWAS grant: 2019_19-169 RG/MATHS/AS_I.

\section{References}

[1] Gilpin, M. E., Ayala, F. J., Global models of growth and competition, Proc Natl Acad Sci USA, 70(12): 3590-3593 (1973).

[2] Protter, M. H., Weinberger, H. F., Maximum Principles in Differential Equations, Prentice Hall, Inc., Englewood Cliffs, N.J. (1967).

[3] Smith, H. L., Monotone Dynamical Systems: An Introduction to the Theory of Competitive and Cooperative Systems, American Mathematical Society, Providence, RI, 41 (1995).

[4] Gopalsamy, K., Exchange of equilibria in two species Lotka-Volterra competition models, J. Austral. Math. Soc. (Series B), 24(2): 160-170 (1982).

[5] Sun, J., Zhang, D., Wu, B., Qualitative properties of cooperative degenerate Lotka-volterra model, Advances in Difference Equations, 281 (2013).

[6] Hsu, S. B., Smith, H. L., Waltman, P., Competitive exclusion and coexistence for competitive systems on ordered Banach spaces, Trans. Amer. Math. Soc., 348(10): 4083-4094 (1996).

[7] Dancer, E. N., Positivity of maps and applications, Topological Nonlinear Analysis, Chapter Part of the Progress in Nonlinear Differential Equations and Their Applications book series (15), 303-340, (1995).

[8] Lam, K. Y., Munther, D., A remark on the global dynamics of competitive systems on ordered Banach spaces, Proc. Am. Math. Soc., 144: 1153-1159 (2015).

[9] Braverman, E., Kamrujjaman, M., Korobenko, L., Competitive spatially distributed population dynamics models: Does diversity in diffusion strategies promote coexistence? Math. Biosci., 264: 63-73 (2015). 
[10] Murray, J. D., Mathematical Biology: I An Introduction, Springer-Verlag (2002).

[11] Sun, G. Q., Mathematical modeling of population dynamics with Allee effect, Nonlinear Dynamics, 85: $1-12(2016)$.

[12] Wittmann, M. J., Stuis, H., Metzler, D., Genetic Allee effects and their interaction with ecological Allee effects, Journal of Animal Ecology, 87(1): 11-23 (2016).

[13] Liu, X., Fan, G., Zhang T., Evolutionary dynamics of single species model with Allee effect, Physica A, 526: 120774 (2019).

[14] Morgan, R., Linearization and Stability Analysis of Nonlinear Problems, Rose-Hulman Undergraduate Mathematics Journal, 16(2): 66-91, Article 5 (2015).

[15] Hirsch, M. W., Smale, S., Devaney, R. L., Differential Equations, Dynamical Systems, and An introduction to Chaos, Elsevier Academic Press, USA (2004).

[16] Guckenheimer, J., Holmes, P., Nonlinear Oscillations, Dynamical Systems, and Bifurcations of Vector Fields, Springer-Verlag, New York (1983).

[17] Huu, K. N., Stability analysis in competition population model, Int. J. Math. Models and Methods in Applied sciences, 6(1): 11-19 (2012).

[18] Hartman, P., A lemma in the theory of structural stability of differential equations, Proceedings of the AMS, 11: 610-620 (1960).

[19] Bicout, D. J., Linear Stability Analysis, EPSP-TIMC, UMR 5525 (2013).

[20] Eberly, D., Stability analysis for systems of differential equations, CA 94042, USA (2008).

[21] Kamrujjaman, M., Interplay of Resource Distributions and Diffusion Strategies for Spatially Heterogeneous Populations, J. Mathematical Modeling, 7(2): 175-198 (2019).

[22] Kamrujjaman, M., Directed vs Regular Diffusion Strategy: Evolutionary Stability Analysis of a Competition Model and an Ideal Free Pair, Differential Equations and Applications, 11(2): 267-290 (2019).

[23] Kamrujjaman, M., Ahmed, A., Ahmed S., Competitive Reaction-Diffusion Systems: Traveling Waves and Numerical Solutions, Advances in Research, 19(6): 1-12 (2019).

[24] Kamrujjaman, M., Weak Competition and Ideally Distributed Populations in a Cooperative Diffusive Model with Crowding Effects, Physical Science International Journal, 18(4): 1-16 (2018).

[25] Kamrujjaman, M., Keya K. N., Global Analysis of a Directed Dynamics Competition Model, J. Advances in Mathematics and Computer Science, 27(2): 1-14 (2018).

[26] Kamrujjaman, M., Dispersal Dynamics: Competitive Symbiotic and Predator-Prey Interactions, J. Advanced Mathematics and Applications, 6: 7-17 (2017).

[27] Perko, L., Differential Equations and dynamical Systems, Springer (2008).

[28] LaSalle, J. P., The stability of dynamical system, Regional Conference Series in Applied Mathematics, SIAM, Philadelphia (1976).

[29] Wiggins, S., Introduction to Applied Nonlinear Dynamical Systems and Chaos, Springer-Verlag, New York (1983). 